\title{
Transient Neonatal Hyperparathyroidism
}

National Cancer Institute

\section{Source}

National Cancer Institute. Transient Neonatal Hyperparathyroidism. NCI Thesaurus.

Code C131032.

Hyperparathyroidism in an infant less than one month a=of age that resolves spontaneously. 